Acta Crystallographica Section D

\section{Biological Crystallography}

ISSN 0907-4449

Jean L. Whittingham, ${ }^{\mathrm{a}} ¥$ Zhang Youshang, ${ }^{\mathbf{b}}$ ‡ Lenka Žáková, ${ }^{c}$ Eleanor J. Dodson, ${ }^{\mathrm{a}}$ Johan $\mathbf{P}$. Turkenburg, ${ }^{a}$ Jens Brange ${ }^{d}$ and G. Guy Dodson ${ }^{\mathrm{a} *}$

${ }^{\text {a } Y o r k ~ S t r u c t u r a l ~ B i o l o g y ~ L a b o r a t o r y, ~ D e p a r t m e n t ~}$ of Chemistry, University of York, Heslington, York YO10 5YW, England, 'b Shanghai Institute of Biochemistry and Cell Biology, Academia Sinica, 320 Yue-Yang Road, Shanghai 200031, People's Republic of China, 'Institute of Organic Chemistry and Biochemistry, Academy of Sciences of the Czech Republic, Flemingovo 2, 16610 Prague, Czech Republic, and ${ }^{\mathbf{d}}$ Brange Consult, Krøyersvej 22C, 2930 Klampenborg, Denmark

₹ These authors contributed equally to this work.

Correspondence e-mail: ggd@ysbl.york.ac.uk

(C) 2006 International Union of Crystallography Printed in Denmark - all rights reserved

\title{
I222 crystal form of despentapeptide (B26-B30) insulin provides new insights into the properties of monomeric insulin
}

Despentapeptide (des-B26-B30) insulin (DPI), an active modified insulin, has been crystallized in the presence of $20 \%$ acetic acid $\mathrm{pH} 2$. A crystal structure analysis to $1.8 \AA$ spacing (space group I222) revealed that the DPI molecule, which is unable to make $\beta$-strand interactions for physiological dimer formation and is apparently monomeric in solution, formed an alternative lattice-generated dimer. The formation of this dimer involved interactions between surfaces which included the B9-B19 $\alpha$-helices (usually buried by the dimer-dimer contacts within the native hexamer). The two crystallographically independent molecules within the dimer were essentially identical and were similar in conformation to T-state insulin as seen in the $\mathrm{T}_{6}$ insulin hexamer. An unusual feature of each molecule in the dimer was the presence of two independent conformations at the B-chain C-terminus (residues B20-B25). Both conformations were different from that of native insulin, involving a $3.5 \AA$ displacement of the B20B23 $\beta$-turn and a repositioning of residue PheB25 such that it made close van der Waals contact with the main body of the molecule, appearing to stabilize the B-chain C-terminus.

\section{Introduction}

In the 1920s the protein hormone insulin was identified as the substance which could reverse the debilitating and ultimately fatal symptoms of diabetes mellitus. Through decades of research on insulin and diabetes, a wealth of knowledge has been accumulated regarding the structure of insulin, its biosynthesis and storage in the pancreas and the role it plays in transmembrane signal transduction. These years of study, however, have failed to reveal the precise mechanism by which insulin binds to and activates its cognate cell-surface receptor. Indeed, there is much discussion about which of the many observed conformations of insulin is the 'active' form.

The first crystal structure of insulin, solved in 1969, contained insulin hexamers consisting of three identical dimers aggregated around two zinc ions (Adams et al., 1969; Baker et al., 1988; Fig. 1). The metal ions coordinated the B10 His side chains of the three dimers. In each monomer of the dimer, the $\mathrm{A}$ and $\mathrm{B}$ chains formed a compact molecule, comprising two $\alpha$-helices in the A chain and one $\alpha$-helix and two extended chain regions in the B chain, later known as the T state (Kaarsholm et al., 1989). The discovery of a second hexameric form of insulin revealed inherent flexibility in the insulin molecule. In the presence of a high chloride ion concentration, three of the B-chain N-termini (residues B1B8) adopted a frayed $\alpha$-helical conformation, later termed the $\mathrm{R}^{\mathrm{f}}$ state (Bentley et al., 1976; Ciszak et al., 1995; Whittingham et al., 1995). When phenol was included in the crystallization, all six B-chain $\mathrm{N}$-termini adopted a fully $\alpha$-helical conforma-
Received 2 December 2005 Accepted 24 February 2006

PDB Reference: despentapeptide insulin, 2ceu, r2ceusf. 
tion termed the R state (Derewenda et al., 1989). Insulin has also been analysed crystallographically as a dimer (Badger et al., 1991; Whittingham et al., 2002) and as a truncated monomer (despentapeptide insulin; Bi et al., 1983). Studies on monomeric insulin in solution using nuclear magnetic resonance (Weiss et al., 1989; Ludvigsen et al., 1994; Olsen et al., 1996) have revealed a molecular conformation very similar to the $\mathrm{T}$ state seen in hexameric insulin crystal structures. When viewed as a whole, these structural studies reveal some variations in the relative positions of the $\mathrm{A}$ - and $\mathrm{B}$-chain $\alpha$-helices and flexibility in the conformations of the B-chain $\mathrm{N}$ and C-termini.

Ideas that $\mathrm{T}$-state insulin could be the active conformation of insulin have been undermined in the light of data from chemically modified insulins such as the essentially inactive A1-B29 cross-linked insulin, which is structurally identical to the native hormone (Derewenda et al., 1991). This and other observations suggest that the B-chain C-terminus undergoes a conformational change during receptor binding (Nakagawa \& Tager, 1987; Mirmira \& Tager, 1989). Indeed, alanine scanning has highlighted residues important in receptor binding, which include several revealed by the displacement of the B-chain Cterminus (Kristensen et al., 1997). While residues B26-B30 can be cleaved from the molecule to produce DPI with no significant effect on biological activity (Fischer et al., 1985; Bi et al., 1983), residues PheB24 and PheB25 are considered to

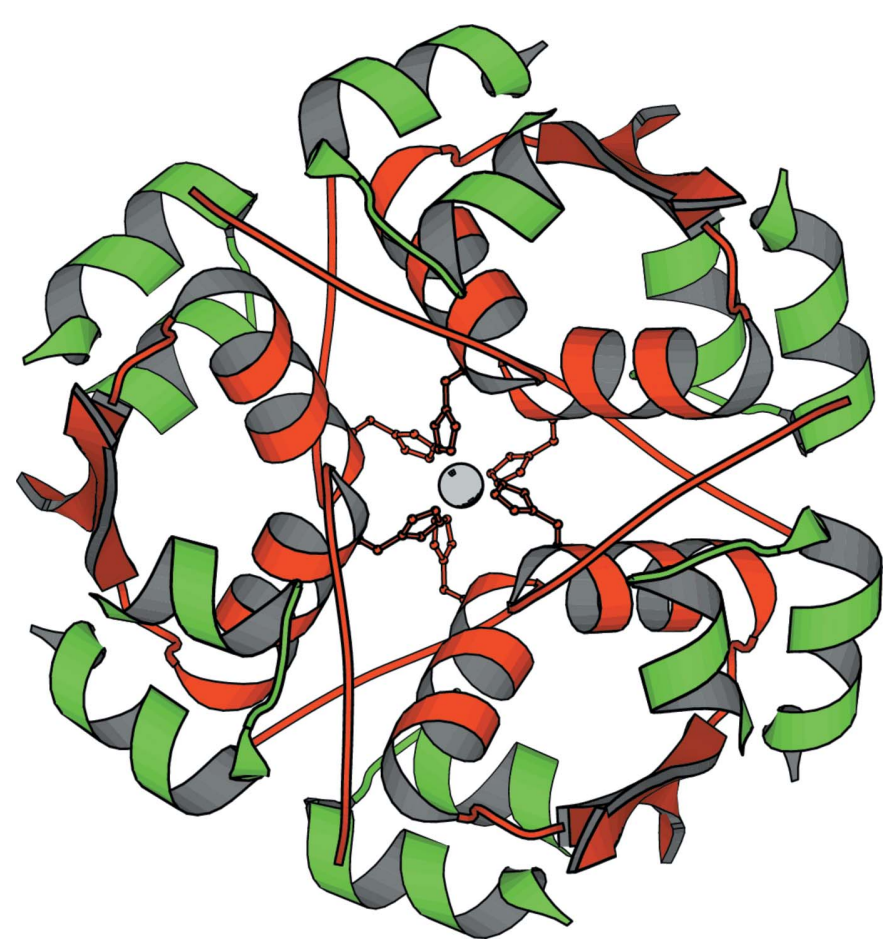

Figure 1

Ribbon diagram of the $2-\mathrm{Zn}$ insulin hexamer viewed down the crystallographic threefold axis. Residues, which are coloured according to chain type (A chain in green, B chain in red), are represented by their $\mathrm{C}^{\alpha}$ atoms only, except for residues HisB10 which coordinate to the zinc ions (grey sphere). This figure was produced using BobScript (Esnouf, 1997). have direct interactions with the receptor (Nakagawa \& Tager, 1986; Mirmira \& Tager, 1989).

In the present study, we wished to investigate the conformation of the insulin monomer in the absence of the structural restraints imposed by physiological dimer and hexamer formation. Initial experiments to crystallize native insulin as a monomer in the presence of acetic acid were apparently successful (Zhang et al., 2002). However, it proved impossible to reproduce these crystals in later experiments and further studies showed that the earlier crystals were DPI. Analysis of the insulin sample used in the original experiments showed that it was substantially contaminated with DPI which evidently preferentially crystallized. The difficulty in obtaining native insulin crystals is itself interesting and is consistent with intrinsic mobility at the surfaces (for example, residues B23B30) which are engaged in forming a lattice. The analysis of DPI crystals grown in $20 \%$ acetic acid was undertaken to investigate whether the molecule's conformation was affected under these extreme conditions.

\section{Materials and methods}

\subsection{Materials}

Human insulin was donated by Novo Nordisk A/S (Denmark). All other reagents, of Analar grade, were purchased from Sigma-Aldrich Fine Chemicals, UK.

\subsection{Preparation of despentapeptide insulin (des-B26-B30; DPI) by pepsin digestion}

Prior to pepsin digestion of human insulin, residual zinc ions (left over from the insulin purification process) were removed using the method of Carpenter (1958). $100 \mathrm{mg}$ human insulin was dissolved in $7 \mathrm{ml} 0.25 \mathrm{M} \mathrm{HCl}$, to which was then added $100 \mathrm{ml}$ cold acetone. This solution was left for $24 \mathrm{~h}$ at $277 \mathrm{~K}$. The solution was then centrifuged at $2000 \mathrm{~g}$ for $20 \mathrm{~min}$ to pellet the precipitated insulin. To check that all of the insulin had been extracted during this process, $50 \mathrm{ml}$ cold acetone was added to $20 \mathrm{ml}$ supernatant. If precipitation reoccurred, cold acetone was added to the whole supernatant and the solution was left for a further $4 \mathrm{~h}$ before repeating the centrifugation step. The precipitated insulin was then dissolved in $10 \mathrm{ml}$ $10 \%(v / v)$ acetic acid and filtered. Finally, the solution was diluted to $5 \%(v / v)$ acetic acid and lyophilized. The yield of zinc-free insulin was $95-97 \%$. If zinc ions are not removed from the preparation, the yield of DPI is reduced.

The pepsin digestion was also carried out according a preestablished protocol (Gattner, 1975). $10 \mathrm{mg}$ zinc-free insulin was dissolved in $1 \mathrm{ml}$ acidified water (adjusted to $\mathrm{pH} 2.3$ using $0.1 \mathrm{M} \mathrm{HCl}$ ) and a solution of pepsin was prepared by dissolving $1 \mathrm{mg}$ of the enzyme in $200 \mu \mathrm{l}$ of the acidified water. Both solutions were cooled to $277 \mathrm{~K}$ on ice. To initiate the digestion, $50 \mu \mathrm{l}$ of the pepsin solution was added to the zinc-free insulin solution, giving an insulin:enzyme molar ratio of 240:1. The reaction mixture was kept at $277 \mathrm{~K}$ for $2 \mathrm{~h}$, during which time $1 \mu \mathrm{l}$ fractions were taken every $10 \mathrm{~min}$, each one being quenched with $9 \mu \mathrm{l}$ of a high-pH buffer $(40 \% 50 \mathrm{mM}$ Tris- $\mathrm{HCl}$ 
pH 8.3, 60\% ethanol). These samples were analysed by nonreducing PAGE in order to check the course of the reaction, which was established to be complete after $1 \mathrm{~h}$, although continuing the incubation did not cause any further digestion. Once the digestion was complete, the whole solution was quenched with $9 \mathrm{ml}$ of the high-pH buffer and then lyophilized.

The DPI was subsequently separated from residual zincfree insulin using RP-HPLC with a Waters (Milford, MA, USA) system comprising a 717 autosampler, 600 MS system controller and 996 photodiode-array detector. Instrument control and data processing were performed using Waters Millenium 2010 software. 70-100 $\mu \mathrm{g}$ DPI was dissolved in $10 \%(v / v)$ acetic acid and isolated on a Vydac 218 TP54 column using gradient elution with acetonitrile $(\mathrm{ACN})$ in water containing $0.1 \%$ TFA $(0 \mathrm{~min} / 8 \%$ ACN, $1 \mathrm{~min} / 32 \% \mathrm{ACN}, 21 \mathrm{~min} / 40 \% \mathrm{ACN}$, $23 \mathrm{~min} / 72 \% \quad \mathrm{ACN}, \quad 25 \mathrm{~min} / 72 \% \quad \mathrm{ACN}$, $25.1 \mathrm{~min} / 8 \% \mathrm{ACN}$ ) at a flow rate of $1 \mathrm{ml} \mathrm{min}{ }^{-1}$. Analysis of the resulting flowthrough, monitored at 258 and $276 \mathrm{~nm}$, showed that zinc-free insulin and DPI ran as separate peaks, having retention times of 9.8 and $10.7 \mathrm{~min}$, respectively. Purified DPI fractions were collected manually and then lyophilized.

\subsection{Crystallization and data collection}

Crystals were grown using the hangingdrop vapour-diffusion method. Each drop consisted of a 50:50 mixture of protein solution [20 $\mathrm{mg} \mathrm{ml}^{-1} \mathrm{DPI} /$ insulin dissolved in $20 \%(v / v)$ acetic acid] and reservoir solution $[20 \%(v / v)$ acetic acid, 0.025-0.050 M sodium sulfate $\mathrm{pH}$ 2]. Crystals grew overnight to a size of approximately $0.01 \mathrm{~mm}^{3}$. A single crystal was vitrified at $120 \mathrm{~K}$ in a cryoprotectant solution consisting of $60 \%(v / v)$ reservoir solution and $40 \%(v / v)$ glycerol prior to data collection on station ID14-2 $(\lambda=0.93300 \AA)$ at the ESRF, Grenoble, France. A $1.80 \AA$ data set was collected by means of a single sweep, with an oscillation angle of $1.0^{\circ}$ per image. The data were processed using $D E N Z O$ (v.1.96.3) and $S C A L E P A C K$ (v.1.96.2) from the $H K L$ package (Otwinowski \& Minor, 1997) and SCALEPACK2MTZ, TRUNCATE and $C A D$ from the $C C P 4$ suite (Collaborative Computational Project, Number 4, 1994). Data-processing statistics are shown in Table 1.

\subsection{Structure solution and refinement}

A monomeric subunit of the 2-Zn insulin hexamer (Baker et al., 1988) was taken as a starting model for molecular replacement, which was carried out using AMoRe (Navaza, 1994) as implemented in the CCP4 suite (Collaborative Computational Project, Number 4, 1994). The rotation and translation parameters were easily identified and provided a useful model for refinement of the DPI structure. This was carried out using maximum-likelihood minimization implemented in REFMAC (Murshudov et al., 1997), with $5 \%$ of the total data being excluded from the refinement for the purpose of $R_{\text {free }}$ calculations. Intermittent manual rebuilding was carried out using the $X$-AUTOFIT facility in $Q U A N T A$ (Oldfield, 2001). Final refinement statistics are listed in Table 1.
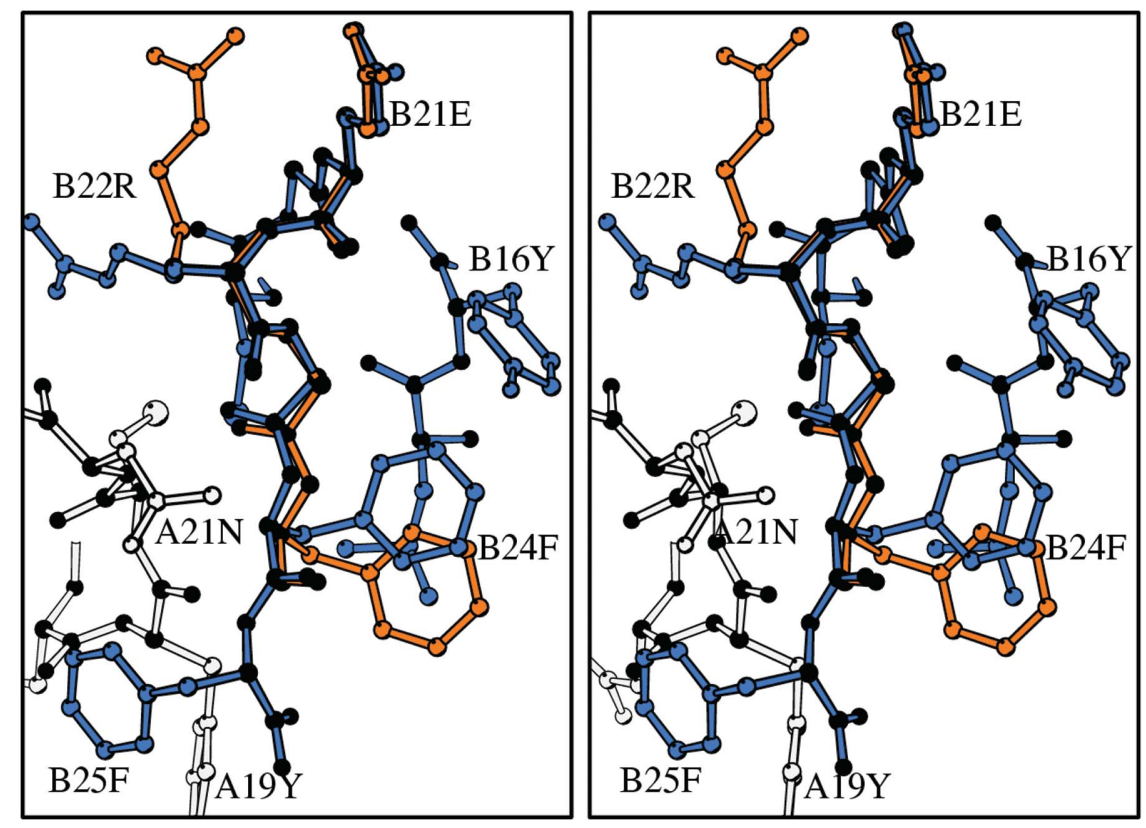

Figure 2

Stereoview ball-and-stick diagram showing the two conformational states at the B-chain C-terminus of DPI_HAc. Residues are coloured according to chain type, with the A chain in grey and the $\mathrm{B}$ chain in blue and orange used to distinguish the two conformers of residues B20-B24. Main-chain atoms are coloured black. Note that the side chain of residue GluB21 and part of that of residue $\mathrm{ArgB} 22$ are disordered in the crystal structure but have been included in the figure for clarity. This figure was produced using BobScript (Esnouf, 1997).
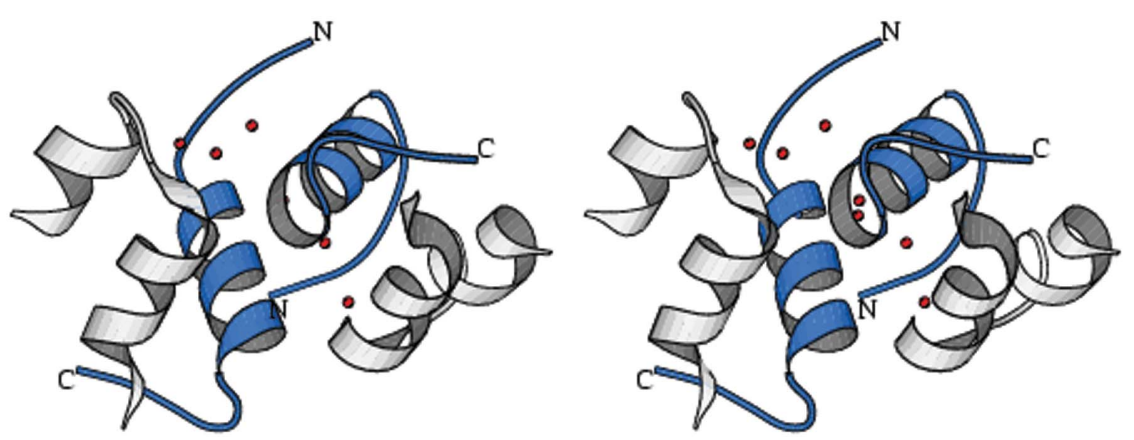

Figure 3

Stereoview ribbon diagram showing the dimeric arrangement of the two molecules of DPI_HAc in the crystallographic asymmetric unit. Residues, which are coloured according to chain type (A chain in grey, B chain in blue), are represented by their $\mathrm{C}^{\alpha}$ atoms only, except for water molecules bound within the dimer, which are shown as red spheres. This figure was produced using BobScript (Esnouf, 1997). 
Table 1

Data-processing and refinement statistics.

Values in parentheses are for the highest resolution shell. Values in square brackets are average ideal values.

\begin{tabular}{ll}
\hline Data-processing statistics & \\
Space group & $I 222$ \\
Unit-cell parameters $(\AA)$ & $a=58.15, b=58.17, c=54.80$ \\
Diffraction limits $(\AA)$ & $25.00-1.80(1.83-1.80)$ \\
No. of unique reflections & 9014 \\
Completeness of data $(\%)$ & $98.2(94.5)$ \\
$R_{\text {sym }}(\%)$ & $3.8(7.1)$ \\
Multiplicity & $3.9(3.7)$ \\
$I / \sigma(I)$ & $31.3(17.5)$ \\
$B_{\text {iso }}\left(\AA^{2}\right)$ & 20.33 \\
Refinement statistics & \\
$R_{\text {cryst }}(\%)$ & 17.38 \\
$R_{\text {free }}(\%)$ & 21.08 \\
R.m.s.d. bond lengths $(1-2)(\AA)$ & $0.016[0.021]$ \\
R.m.s.d. angles $\left({ }^{\circ}\right)$ & $1.490[1.972]$ \\
R.m.s.d. chiral volumes $\left(\AA^{3}\right)$ & $0.085[0.200]$ \\
$\sigma_{\mathrm{A}}$ estimated coordinate error $(\AA)$ & 0.32 \\
Mean temperature factors $\left(\AA^{2}\right)$ & 19.09 \\
Allowed Ramachandran angles $(\%)$ & \\
$\quad$ Most favoured regions & 92.1 \\
Additionally allowed regions & 7.9
\end{tabular}

\section{Results}

The refined model consists of two molecules of DPI, 65 water molecules and three sulfate ions (one at $1 / 3$ occupancy) per asymmetric unit (Table 1). The solvent content of the crystal was calculated to be $45 \%$, with an associated Matthews coefficient of $2.2 \AA^{3} \mathrm{Da}^{-1}$ (Matthews, 1968). The two protein molecules are related by an almost exact non-crystallographic twofold symmetry element. The structure was initially solved and refined in space group I 422 and erroneously published as native insulin (Zhang et al., 2002). The absence of detectable electron density for residues B26-B30 was explained by the assumption that residues $\mathrm{B} 26-\mathrm{B} 30$ were disordered, something not unexpected in the monomer. Although there were no problems with the refinement overall, there were difficulties in resolving two closely related conformations of the B-chain C-terminus (residues B20-B25) located near the crystallographic fourfold axis. Following careful analysis of the diffraction data, it was decided that the space group was $I 222$. Consequently, a new set of data were collected to allow for the lower symmetry. Once the structure had been re-solved as DPI and re-refined, the electron density in the vicinity of residues B20-B25 was sufficiently informative for the two conformations to be built separately. At the conclusion of the refinement, the crystallographic $R$ factor stood at $0.1738\left(R_{\text {free }}=\right.$ 0.2108). Other refinement statistics are listed in Table 1.

The two DPI molecules (termed DPI_HAc to distinguish them from other DPI structures) are essentially identical in conformation, with an r.m.s. difference of $0.10 \AA$ calculated on all main-chain atoms. Each molecule comprises three $\alpha$-helices (residues A2-A8, A13-A20 and B9-B19), a loop (residues $\mathrm{A} 9-\mathrm{A} 12)$ and extended B-chain $\mathrm{N}$ - and C-termini (residues $\mathrm{B} 1-\mathrm{B} 8$ and $\mathrm{B} 20-\mathrm{B} 25)$. Most of the residues are well ordered with single occupancies, although residue PheB1 is completely disordered owing to displacement by adjacent DPI_HAc dimers in the crystal. At the B-chain C-terminus, residues B20-B24 each occupy two distinct, albeit overlapping, conformations (Fig. 2). The side chains of $\operatorname{ArgB} 22$, although poorly defined in the electron-density maps, have different conformations, while those of residue GluB21 are not visible at all (as is often the case in insulin structures). The two conformations of PheB24 are the most interesting, one being stabilized by an intramolecular ring-stacking interaction with the side chain of TyrB16 and the other occupying space vacated by the enzymatic removal of residues B26-B30 (Fig. 2). Residue PheB25 occupies only one position and is stabilized principally by an intramolecular stacking interaction with the side chain of residue TyrA19. This interaction appears to be quite strong: the electron density for this residue was well defined in the early stages of the refinement when it was difficult to pinpoint the positions of the other residues at the B-chain C-terminus. Furthermore, for residue PheB25 the average temperature factor for the side-chain atoms $\left(28.0 \AA^{2}\right)$ is somewhat less than that of the main-chain atoms $\left(34.4 \AA^{2}\right)$. Generally, the average temperature factor for all of the atoms of residues $\mathrm{B} 21-\mathrm{B} 25\left(31.0 \AA^{2}\right)$ is higher than that for all of the atoms in the rest of the molecule $\left(19.5 \AA^{2}\right)$.

The contacting surfaces of the two crystallographically independent DPI_HAc molecules lie perpendicular to one another and are closely associated through van der Waals contacts between two complementary surfaces which include residues CysA11, SerA12, LeuA13, LeuA16, ValB2, AsnB3, GlnB4, HisB5, LeuB6, HisB10, GluB13, AlaB14, LeuB17 and ValB18 (Fig. 3). The most hydrophobic of these residues cluster in the centre of the dimer, while eight water molecules buried at the monomer-monomer interface hydrogen bond across the interface, providing extra stability to the dimer. These contact surfaces are the same as those involved in dimer-dimer interactions in the $\mathrm{T}_{6}$ insulin hexamer (Baker et al., 1988), although the surface packing in the DPI_HAc dimer is somewhat different (Fig. 4). On the surface of the DPI_HAc dimer, an $\mathrm{HSO}_{4}^{-}$ion located at the end of each A-chain $\mathrm{N}$ terminal $\alpha$-helix makes hydrogen bonds with the amide $\mathrm{N}$ atoms of residues GlyA1 and GlnA4 (Fig. 5). This anion is not directly involved in crystal contacts, but facilitates crystal formation by neutralizing the charge at the A-chain N-termini, which are located within $7.2 \AA$ of each other in the crystal and possess considerable positive charge (Whittingham et al., 2002). A third partially occupied $\mathrm{HSO}_{4}^{-}$ion-binding site is situated at the intersection of two crystallographic twofold axes. Here, the sulfate ion is surrounded by 12 water molecules and mediates crystal contacts between four DPI_HAc dimers. Given the high acetic acid content of the crystallization medium, it was considered that acetic acid molecules might be making direct ordered interactions with the protein. No acetic acid molecules could be located in the electron-density maps, however.

Comparisons of a DPI_HAc molecule with two different crystal forms of native insulin, taken from the $T_{6}$ insulin hexamer (crystallized at approximately $\mathrm{pH} 8$ ) and the low-pH insulin dimer (Baker et al., 1988; Whittingham et al., 2002), show that the $\alpha$-helical cores of the molecules are very similar, 
with significant differences occurring only at the B-chain $\mathrm{N}$ and C-termini (Figs. $6 a$ and $6 b$ ). The B-chain N-terminus of the $\mathrm{T}_{6}$ insulin monomeric subunit curves round the molecule, whereas those in both the low-pH insulin dimer and DPI_HAc extend outwards in a well defined conformation. Towards the B-chain C-terminus, a conserved $\beta$-turn (residues B20-B23) has shifted in position by some $3.5 \AA$ in DPI_HAc, resulting in the loss of hydrogen bonds between the carboxy-terminus of residue AsnA21 and the NE atom of residue ArgB22. The distortion of the $\beta$-turn results in the loss of the main-chain $\mathrm{B} 19 \mathrm{O}-\mathrm{B} 22 \mathrm{~N}$ hydrogen bond, although the $\mathrm{B} 20 \mathrm{O}-\mathrm{B} 23 \mathrm{~N}$ hydrogen bond is conserved. A comparison was also made with a previously reported crystal form of DPI (Bi et al., 1983). The two DPI molecules were similar in overall conformation, but differed significantly in the relative positions of the core $\alpha$-helices (Fig. 6c). It was concluded from this that the DPI structure reported by $\mathrm{Bi}$ et al. (1983) has suffered some distortions owing to the compact nature of this crystal form (solvent content $28 \%$ ) and therefore further comparison of these structures was not considered to be useful.

\section{Discussion}

Analysis of the DPI_HAc molecule shows that the arrangement of the three $\alpha$-helices is identical to that in T-state insulin from the 2-Zn insulin hexamer (Fig. $6 a$ ) and very similar to those in NMR structures of monomeric insulins (Weiss et al., 1989; Ludvigsen et al., 1994; Olsen et al., 1996). This indicates that the core of the DPI_HAc molecule had not been unduly distorted by crystal packing forces, although peripheral parts of the molecule have been. For instance, the displacement of residue PheB24 arises from a displacement of the side chain of nearby residue TyrB16 by the side chain of residue GlnB4 of an adjacent monomer. Similarly, the deformation of the B20B23 $\beta$-turn arises from the displacement of the side chain of residue ArgB22 by residue SerB9 of an adjacent dimer, leading to the loss of the structurally important hydrogen bond between the carboxy-terminus of residue AsnA21 and the NE atom of residue ArgB22.

The influence of crystal packing forces on the B-chain Cterminus is a testament to its inherent flexibility. This characteristic is known to be an important factor in receptor binding, although to what degree this part of the molecule moves during this process is still unknown. It is impossible to say if the unusual conformation of residues B20-B25 in DPI_HAc is representative of the conformation of insulin as it binds to its receptor. However, the interaction of residue PheB25 with the main body of the DPI molecule is favourable and demonstrates the capacity of the hydrophobic side chains of the B-chain C-terminus to stabilize this part of the molecule in the absence of physiological dimer formation. Indeed, NMR studies reveal that in $20 \%$ acetic acid the B-chain C-terminus of GlyB24 mutant insulin becomes partially unfolded (Hua et al., 1991; Ludvigsen et al., 1998). Hence, it seems likely that prior to or during the receptor-binding process residues PheB24 and PheB25 play a role in stabilizing the B-chain C- terminus, perhaps maintaining it in the correct position for interaction with the receptor.

As so often with insulin and insulin-like molecules, the aggregation state of the molecule provides further insights. In the $I 222$ crystal form, DPI forms a weak dimer stabilized by interactions between the B-chain $\alpha$-helices and a number of well ordered water molecules. At the $\mathrm{pH}$ of crystallization, $\sim \mathrm{pH} 2$, there will be a surplus of positive charges on the protein which could affect the way the protein aggregates. In fact, only two residues, HisB10 from each of the two molecules, are present at the monomer-monomer interface, their positively charged side chains pointing towards the outside of the dimer in close proximity to one another. In contrast, the equivalent (neutral) side chains in a pH 5.9 crystal structure of desheptapeptide (B24-B30) insulin DHPI (which forms the same sort of dimer as DPI_HAc; Bao et al., 1997) have a better special arrangement, with one of the side chains accommodated at the monomer-monomer interface. Further comparison of these two structures shows that the conformations of residue GluB13 are also dependent on $\mathrm{pH}$. In DPI_HAc they are neutral and run alongside hydrophobic side chains at the monomer-monomer interface, whilst in DHPI they carry charge and point away from the interface. Hence, the $\mathrm{pH}$ of crystallization seems to affect the conformations of certain side chains but does not affect the overall aggregation of the protein. Rather, it appears that this sort of dimerization is favoured when physiological dimer formation is prevented, in these cases by truncation of the molecule. In theory, there is no reason why full-length insulin could not form this alternative dimer. Crystallization of this species, however, might have proved difficult owing to the extended flexible B-chain C-terminus.

As a final comment, it is worth considering the parallels between the crystallization of DPI in $20 \%$ acetic acid and insulin amyloid formation. Insulin and insulin-like molecules (especially DPI) have the capacity to fibrillate when subjected to heat and agitation (Waugh et al., 1953). The most ordered fibres are obtained in mineral acids where, with agitation and heat, the molecule is monomeric and counterions such as chloride and sulfate encourage elongation and bunching of protofibres (Whittingham et al., 2002). Fibres are also obtained in acetic acid at elevated temperatures, although the fibrillation process is much slower. The crystallization of DPI_HAc mimics the fibrillation process in so far as both events involve the aggregation of molecules under acidic conditions, employing counterions to assist in the aggregation process. It is likely that the amyloid protofibres, such as the DPI_HAc dimer, contain a core of hydrophobic residues surrounded by polar and charged residues. The crucial difference between these two processes lies in the degree to which the protein is folded in each case. There is an intellectual case for a model for amyloid fibre in which the protein is largely unfolded and forms a periodic structure based on $\beta$-strand interactions. There is evidence for this, but it remains inconclusive (Nielsen et al., 2001). A recent report of an epitope present in the prion molecule is relevant to this argument. The epitope, which sits between two disulfide-linked $\alpha$-helices and is common to $\operatorname{PrP}^{\mathrm{C}}$ 


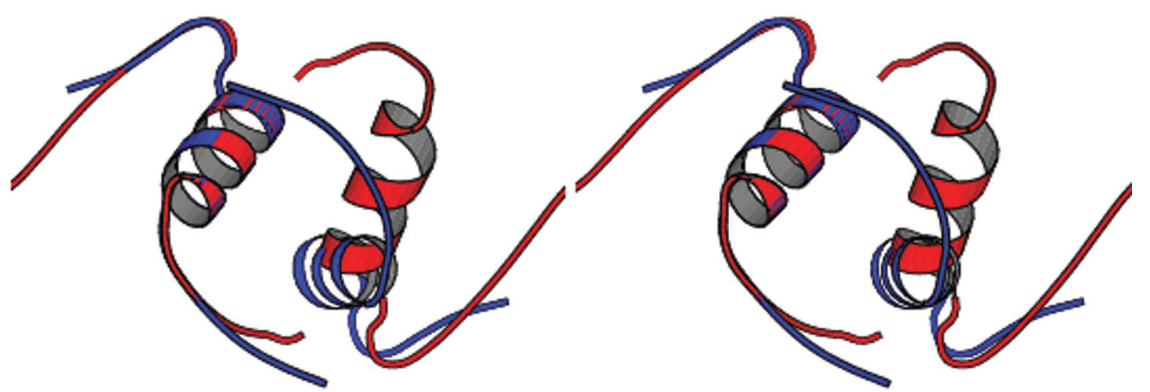

Figure 4

Stereoview ribbon diagram comparing the packing of the two DPI_HAc molecules in the asymmetric unit with the packing of two molecules at the dimer-dimer interface in the $\mathrm{T}_{6}$ insulin hexamer. The B chains of the DPI_HAc dimer (in blue) are superimposed upon the B chains of two adjacent molecules from two different dimers in the 2-Zn insulin hexamer (in red). The superposition was made by the alignment of only the left-hand B9-B19 $\alpha$-helix in each pair of molecules so as to illustrate the different packing arrangements of the two pairs of molecules. This figure was produced using BobScript (Esnouf, 1997).
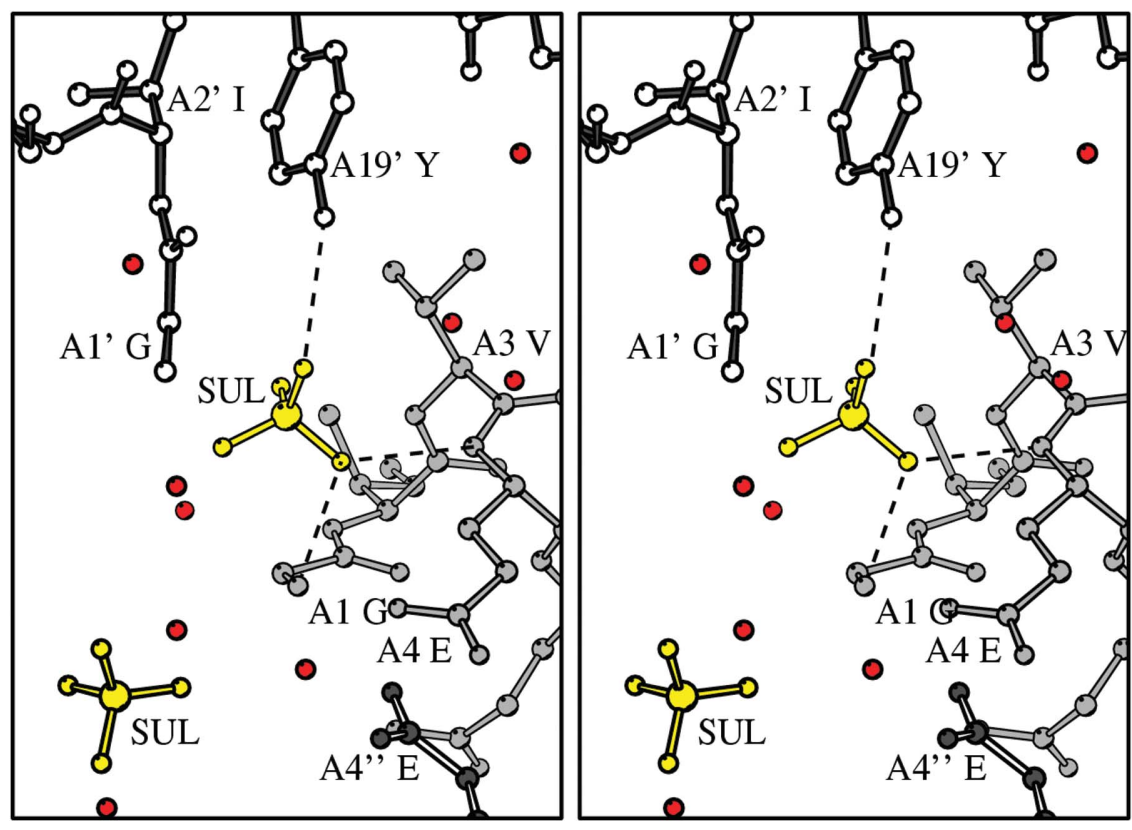

Figure 5

Stereoview ball-and-stick diagram showing the two $\mathrm{HSO}_{4}^{-}$ion-binding sites in the DPI_HAc crystal structure. $\mathrm{HSO}_{4}^{-}$ions are coloured yellow, adjacent DPI_HAc dimers in the crystal are coloured either grey or black with white atoms and water molecules are represented by red spheres. Hydrogen bonds between one of the $\mathrm{HSO}_{4}^{-}$ions and atoms of the A-chain $\mathrm{N}$-terminus are shown as dashed lines. This figure was produced using BobScript (Esnouf, 1997). (the prion cellular and globular state) and $\mathrm{PrP}^{\mathrm{Sc}}$ (the prion fibrous state) structures, is strong evidence that $\alpha$-helices can be present in amyloid fibre (Eghiaian et al., 2004). In the case of insulin, the relatively mild conditions of fibre formation are consistent with preservation of some $\alpha$ helical structure given that these are linked together by disulfide bonds. In this respect, the amphipathic nature of the B9-B19 $\alpha$ helix is particularly well suited to act as a nucleus for alternative fibre-forming interactions.

We wish to thank Angela Squier and Brendan Keely for assistance with the protein purification, and Anthony Wilkinson and Garib Murshudov for useful discussions. We would also like to acknowledge colleagues at Novo Nordisk A/S (Denmark) who provided both funding and insulin material. X-ray data were collected at the European Synchrotron Radiation Facility in Grenoble, station ID14-2. The Royal Society is thanked for providing funds for ZYS to visit York. The work of LZ was supported by Research Project Z4 0550506.

\section{References}

Adams, M. J., Blundell, T. L., Dodson, E. J., Dodson, G. G., Vijayan, M., Baker, E. N., Harding, M. M., Hodgkin, D. C., Rimmer, B. \& Sheat, S. (1969). Nature (London), 224, 491495.

Badger, J., Harris, M. R., Reynolds, C. D., Evans, A. C., Dodson, E. J., Dodson, G. G. \& North, A. C. T. (1991). Acta Cryst. B47, 127-136.

Baker, E. N., Blundell, T. L., Cutfield, J. F., Cutfield, S. M., Dodson, E. J., Dodson, G. G., Crowfoot Hodgkin, D. M., Hubbard, R. E., Isaacs, N. W., Reynolds, C. D., Sakabe, K.,

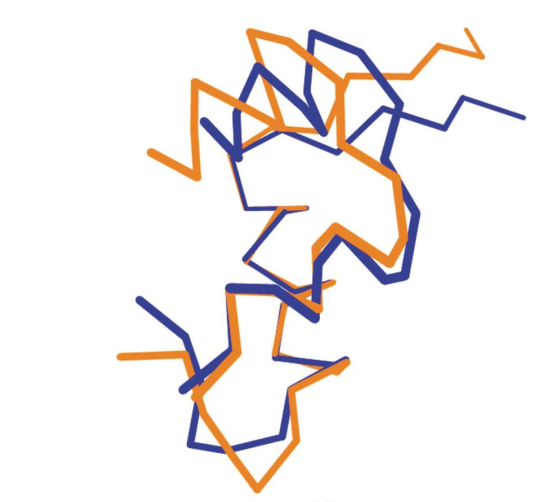

(c)

Figure 6

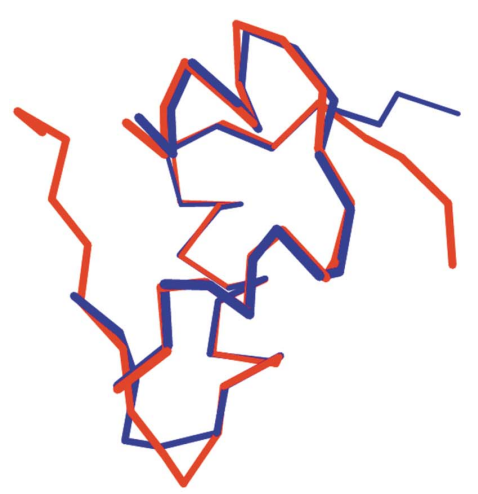

(a)

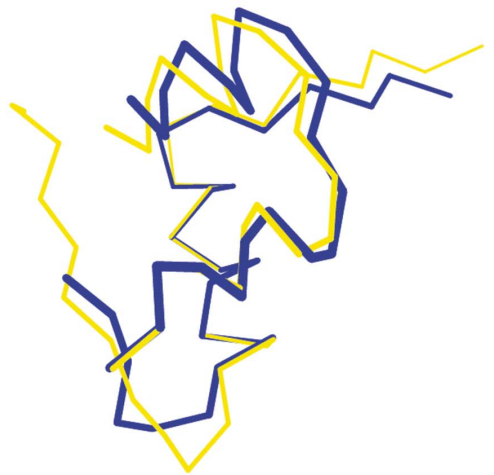

(b) $\mathrm{C}^{\alpha}$-trace comparisons of the DPI_HAc molecule (blue) with $(a)$ molecule 1 of $\mathrm{T}_{6}$ insulin (red), $(b)$ molecule 1 of the low-pH orthorhombic insulin
(yellow) and $(c)$ DPI (orange; Bi et al., 1983). Molecules were superimposed on the B9-B19 $\alpha$-helices using the CCP4 suite (Collaborative Computational Project, Number 4, 1994). This figure was produced using BobScript (Esnouf, 1997) and Raster3D (Merritt \& Bacon, 1997). 
Sakabe, N. \& Vijayan, N. M. (1988). Philos. Trans. R. Soc. London Ser. B, 319, 369-456.

Bao, S.-J., Xie, D.-L., Zhang, J.-P., Chang, W.-R. \& Liang, D.-C. (1997). Proc. Natl Acad. Sci. USA, 94, 2975-2980.

Bentley, G., Dodson, E., Dodson, G., Hodgkin, D. \& Mercola, D. (1976). Nature (London), 261, 166-168.

Bi, R.-C., Dauter, Z., Dodson, E., Dodson, G., Gordiano, F., Hubbard, R. \& Reynolds, C. (1983). Proc. Indian Acad. Sci. (Chem. Sci.), 92, 473-483.

Carpenter, F. H. (1958). Arch. Biochem. Biophys. 78, 539-545.

Ciszak, E., Beals, J. M., Frank, B. H., Baker, J. C., Carter, N. D. \& Smith, G. D. (1995). Structure, 3, 615-622.

Collaborative Computational Project, Number 4 (1994). Acta Cryst. D50, 760-763.

Derewenda, U., Derewenda, Z., Dodson, E. J., Dodson, G. G., Reynolds, C. D., Smith, G. D., Sparks, C. \& Swenson, D. (1989). Nature (London), 338, 594-596.

Derewenda, U., Derewenda, Z., Dodson, E. J., Dodson, G. G. \& Xiao, B. (1991). J. Mol. Biol. 220, 425-433.

Eghiaian, F., Grosclaude, J., Lesceu, S., Debey, P., Doublet, B., Tréguer, E., Rezaei, H. \& Knossow, M. (2004). Proc. Natl Acad. Sci. USA, 101, 10254-10259.

Esnouf, R. M. (1997). J. Mol. Graph. 15, 132-134.

Fischer, W. H., Saunders, D., Brandenburg, D., Wollmer, A. \& Zahn, H. (1985). Biol. Chem. Hoppe-Seyler, 366, 521-525.

Gattner, H. G. (1975). Hoppe-Seyler's Z. Physiol. Chem. 356, 13971404.

Hua, Q. X., Shoelson, S. E., Kochoyan, M. \& Weiss, M. A. (1991). Nature (London), 354, 238-241.

Kaarsholm, N. C., Hui-Chong, K. \& Dunn, M. F. (1989). Biochemistry, 28, 4427-4435.

Kristensen, C., Kjeldsen, T., Wilberg, F. C., Schäffer, L., Hach, M., Havelund, S., Bass, J., Steiner, D. F. \& Andersen, A. S. (1997). J. Biol. Chem. 272, 12978-12983.
Ludvigsen, S., Olsen, H. B. \& Kaarsholm, N. C. (1998). J. Mol. Biol. 279, 1-7.

Ludvigsen, S., Roy, M., Thøgersen, H. \& Kaarsholm, N. C. (1994). Biochemistry, 33, 7998-8006.

Matthews, B. W. (1968). J. Mol. Biol. 33, 491-497.

Merritt, E. A. \& Bacon, D. J. (1997). Methods Enzymol. 277, 505524.

Mirmira, R. G. \& Tager, H. S. (1989). J. Biol. Chem. 264, 63496354.

Murshudov, G. N., Dodson, E. J. \& Vagin, A. A. (1997). Acta Cryst. D53, 240-255.

Nakagawa, S. H. \& Tager, H. S. (1986). J. Biol. Chem. 261, 7332-7341.

Nakagawa, S. H. \& Tager, H. S. (1987). J. Biol. Chem. 262, 12054 12058.

Navaza, J. (1994). Acta Cryst. A50, 157-163.

Nielsen, L., Frokjaer, S., Carpenter, J. F. \& Brange, J. (2001). J. Pharm. Sci. 90, 29-37.

Oldfield, T. J. (2001). Acta Cryst. D57, 82-94.

Olsen, H. B., Ludvigsen, S. \& Kaarsholm, N. C. (1996). Biochemistry, 35, 8836-8845.

Otwinowski, Z. \& Minor, W. (1997). Methods Enzymol. 276, 307326.

Waugh, D. F., Wilhelmson, D. F., Commerford, S. L. \& Sackler, M. L. (1953). J. Am. Chem. Soc. 75, 2592-2600.

Weiss, M. A., Nguyen, D. T., Khait, I., Inouye, K., Frank, B. H., Beckage, M., O'Shea, E., Shoelson, S. E., Karplus, M. \& Neuringer, L. J. (1989). Biochemistry, 28, 9855-9873.

Whittingham, J. L., Chaudhuri, S., Dodson, E. J., Moody, P. C. E. \& Dodson, G. G. (1995). Biochemistry, 34, 15553-15563.

Whittingham, J. L., Scott, D. J., Chance, K., Wilson, A., Finch, J., Brange, J. \& Dodson, G. G. (2002). J. Mol. Biol. 318, 479-490.

Zhang, Y., Whittingham, J. L., Turkenburg, J. P., Dodson, E. J., Brange, J. \& Dodson, G. G. (2002). Acta Cryst. D58, 186187. 